\title{
Colecistitis aguda litiásica: aplicación de las Guías de Tokio en los criterios de gravedad
}

\author{
Litiasic acute cholecystitis: application of Tokyo Guidelines in severity grading
}

Alfredo Escartín*, Marta González, Pablo Muriel, Elena Cuello, Ana Pinillos, Maite Santamaría, Helena Salvador y Jorge-Juan Olsina

Servicio de Cirugía General, Institut de Recerca Biomèdica de Lleida Fundació Dr. Pifarré (IRBLleida), Hospital Universitario Arnau de Vilanova, Lleida, España

\section{Resumen}

Antecedentes: La colecistitis aguda litiásica $(C A)$ es una de las urgencias quirúrgicas más frecuentes en nuestro médio. La colecistectomía laparoscópica se considera el tratamiento de elección, aunque sigue sin ser una realidad su práctica generalizada. Objetivo: Analizar la aplicación de las Guías de Tokio en el manejo de la CA y determinar la influencia de la gravedad en el manejo y el pronóstico. Método: Estudio prospectivo, observacional, de pacientes con diagnóstico primario de CA entre 2010 y 2015. Criterios de exclusión: recidiva de CA, CA como diagnóstico secundario, CA alitiásica u otra patología biliar concomitante. Se ha clasificado la gravedad según las Guías de Tokio de 2013. Resultados: Se incluyen 998 CA: 338 (33.9\%) leves, 567 (56.8\%) moderadas y 93 (9.3\%) graves. Se operaron 582 pacientes (58.3\%), y posteriormente 15 precisaron rescate. Complicaciones posoperatorias Dindo-Clavien $\geq 12,6 \%$ : CA leve 3,6\%, CA moderada $12,2 \%$, CA grave 49\% ( $p<0.001)$. Mortalidad global $2 \%$ : CA leve 0\%, CA moderada $0.5 \%, C A$ grave $18 \%(p<0.001)$. Conclusión: La colecistectomía laparoscópica sigue siendo el tratamiento de elección para la CA leve y moderada. En pacientes con CA grave debe valorarse el riesgo-beneficio de la cirugía, dadas las complicaciones y la mortalidad asociadas.

PALABRAS CLAVE: Colecistectomía laparoscópica. Colecistitis aguda. Colecistostomía. Guías de Tokio. Recidiva.

\begin{abstract}
Background: Acute calculous cholecystitis $(A C)$ is one of the most frequent surgical emergencies in our field. Laparoscopic cholecystectomy is considered the treatment of choice, although not sufficiently widespread. Objective: To analyze the application of the Tokyo Guidelines in the management of $A C$ and to determine the influence of the degree of severity on management and prognosis. Method: Prospective, observational study of patients with a primary diagnosis of AC between 2010 and 2015. Exclusion criteria: AC recurrence; $A C$ as a secondary diagnosis; acalculous cholecystitis; concurrent bifiary pathology. Severity was classified according Tokyo 2013 Guidelines. Results: 998 patients were included: 338 (33.9\%) mild $A C, 567(56.8 \%)$ moderate $A C$, and 93 (9.3\%) severe AC. A total of 582 (58.3\%) patients were operated on. Postoperative complications Dindo-Clavien grade $\geq$ II 12.6\%: mild $A C$ 3.6\%; moderate $A C 12.2 \%$; severe $A C 49.0 \%(p<0.001)$. Overall mortality 2\%: mild AC 0\%; moderate AC 0.5\%; severe AC 18.0\% ( $p<0.001)$. Conclusion: Urgent laparoscopic cholecys-
\end{abstract}


tectomy remains the treatment of choice for mild and moderate AC. In patients with severe $A C$, the risks and benefits of surgery should be assessed, given the high degree of complications and associated mortality.

Key words: Laparoscopic cholecystectomy. Acute cholecystitis. Cholecystostomy. Tokyo guidelines. Recurrence.

\section{Introducción}

La colecistitis aguda litiásica (CA) es una de las principales urgencias quirúrgicas, principalmente en la población de edad avanzada debido al progresivo incremento de la esperanza de vida'. Aunque la evolución es habitualmente favorable, puede presentar complicaciones y tiene una mortalidad no despreciable, en función de las características de la enfermedad y del paciente ${ }^{2}$.

Aunque no hemos encontrado metaanálisis concluyentes ${ }^{3}$, la colecistectomía laparoscópica (CL) urgente se considera el tratamiento de elección de la $\mathrm{CA}^{4-10}$, aunque los pacientes no siempre son intervenidos por los riesgos que conlleva ${ }^{11}$; el mal estado general del paciente o la edad se han considerado los principales factores de riesgo ${ }^{12-14}$, si bien el criterio edad avanzada ha ido variando con el tiempo ${ }^{15-18}$. El principal problema de los estudios referidos y de la literatura en general era la falta de un criterio homogéneo que permitiera realizar análisis comparativos entre los resultados y las distintas opciones terapéuticas.

La publicación en $2007^{19}$ (actualizada en $2013^{20}$ y $2018^{21}$ ) de las Guías de Tokio tenía como objetivo unificar los criterios diagnósticos y clasificar a los pacientes en función de unas medidas definidas de gravedad, facilitando la toma de decisiones y el posterior análisis.

El objetivo del presente estudio fue analizar nuestra experiencia en la aplicación de las Guías de Tokio al manejo de la CA, y la influencia que el grado de gravedad tiene en el manejo y el pronóstico de los pacientes.

\section{Método}

Presentamos un estudio clínico observacional sobre la base de datos recogida de forma prospectiva de los pacientes ingresados de forma consecutiva con CA en nuestro centro a partir de 2010 , momento en que se modificó nuestro protocolo de diagnóstico y tratamiento para adaptarlo a las Guías de Tokio. Tras la actualización de estas en 2013 adaptamos de nuevo nuestros protocolos y se revisaron los pacientes incluidos hasta ese momento en la base.
La base incluye datos de todos los pacientes ingresados desde el servicio de urgencias con diagnóstico de primer episodio de CA, entre junio de 2010 yூ diciembre de 2015. Los criterios de exclusión fueron: recidiva de un episodio anterior de CA, CA como

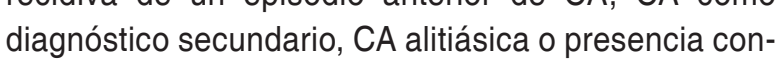
comitante de pancreatitis aguda, colangitis aguda u otra patología biliar (benigna o neoplásica), a excepción de la coledocolitiasis diagnosticada de forma simultánea con la CA. Se recogieron para su posterior análisis las siguientes características: edad, sexo, antecedentes clínicos, antecedentes biliares (pancreatitis aguda, colangitis aguda o ictericia obstructiva), coledocolitiasis simultánea y clasificación ASA (AM̈)erican Society of Anesthesiologists). Consideramos $\mathrm{Co}$ morbilidad significativa los pacientes con grado ÁSA III O IV. El tratamiento inicial fue el decidido eh el momento del ingreso (antibiótico, colecistostomía ecoguiada o colecistectomía). Definimos colecistecttomía precoz como la realizada en las primeraš 48 horas desde el ingreso. Se registraron la duraciónidel tratamiento, los reingresos, la mortalidad y las cormplicaciones (según la escala Dindo-Clavien ${ }^{22}$ ). Lacolecistectomía diferida es la realizada tras el alta del paciente, habitualmente a partir de la sexta semanna. El seguimiento de los pacientes finalizó a los 30 días de la colecistectomía en aquellos que fueron intervenidos (de manera urgente o electiva) o hasta e fallecimiento y a los 24 meses en el resto.

\section{Diagnóstico}

El diagnóstico se realizó en el servicio de urgencias de acuerdo con las Guías de Tokio ${ }^{20}$ basándosê en criterios clínicos, analíticos y radiológicos (Tablä 1). En función del estado del paciente se realizó también tomografía computarizada o resonancia magnética. La gravedad de la CA se clasificó en grado I (levêd, II (moderada) o III (grave) $)^{20}$ (Tabla 2).

\section{Manejo en el servicio de urgencias}

Se inició tratamiento con fluidoterapia, analgesia, omeprazol y antibioticoterapia según el protocolō del hospital. Posteriormente, los pacientes ingresaron en 
Tabla 1. Criterios diagnósticos de la colecistitis aguda según las Guías de Tokio

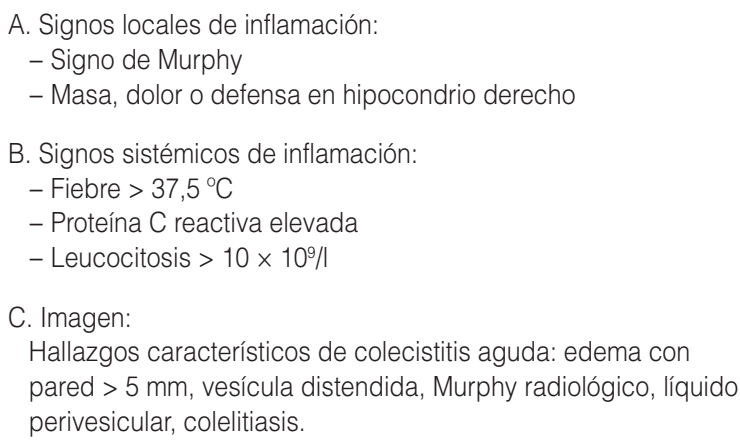

Sospecha diagnóstica: un criterio de $A+$ un criterio de $B$ Diagnós tico definitivo: un criterio de $A+$ un criterio de $B+C$

Tabla 2. Criterios de gravedad de la colecistitis aguda según las Guías de Tokio

- Grado I (leve): no cumple criterios de grado II o grado III

- Grado II (moderada), se asocia a una de las siguientes condiciones:

1) Aumento del recuento leucocitario $\left(>18.000 / \mathrm{mm}^{3}\right)$

2) Masa blanda palpable en el hipocondrio derecho

3) Duración de los síntomas $>72$ horas

4) Marcada inflamación local (colecistitis gangrenosa, absceso perivesicular, absceso hepático, colecistitis enfisematosa, coleperitoneo)

- Grado III (grave), se asocia con disfunción de uno de los siguientes órganos o sistemas:

1) Cardiovascular: hipotensión que requiere tratamiento con dopamina $\geq 5 \mu \mathrm{g} / \mathrm{kg}$ por minuto, o cualquier dosis de epinefrina

2) Neurológica: disminución del nivel de consciencia

3) Respiratoria: $\mathrm{PaO}_{2} / \mathrm{FiO}_{2}<300$

4) Renal: oliguria o creatinina $>2.0 \mathrm{mg} / \mathrm{dl}$

5) Hepática: tiempo de protrombina, INR $>1.5$

6) Hematológica: recuento plaquetario $<100.000 / \mathrm{mm}^{3}$

INR: International Normalized Ratio; $\mathrm{FiO}_{2}$ : fracción de oxígeno en el aire inspirado; $\mathrm{PaO}_{2}$ : presión arterial de oxígeno.

planta de hospitalización para completar el tratamiento. Los pacientes con CA grave se trataron de manera individualizada, con ingreso en la unidad de cuidados intensivos cuando cumplieron los criterios. En casos seleccionados, los pacientes con CA leve fueron atendidos en sus casas por el servicio de hospitalización a domicilio ${ }^{23}$.

El protocolo antibiótico seguido, de acuerdo con las recomendaciones de las guías ${ }^{24-26}$, fue el siguiente: para CA leve, ceftriaxona + metronidazol; para CA moderada, + ceftriaxona-metronidazol (ertapenem en pacientes mayores de 75 años o con factores de riesgo); y para CA grave, piperacilina-tazobactam. En caso de alergia se usó gentamicina + metronidazol en la CA leve-moderada y tigeciclina + amikacina en la CA grave. La duración del tratamiento, si no se realizó colecistectomía, fue de 4-7 días, generalmente hasta la normalización de los leucocitos y la disminución a la mitad de los valores iniciales de la proteína C reactiva; en caso de realizar colecistectomía el tratamiento se suspendió a las 24 horas de la intervención, excepto en los pacientes con CA enfisematosa, necrosis vesicular, perforación o absceso perivesicular, en los que se mantuvo también 4-7 días.

\section{Opciones terapéuticas}

La CL precoz es el tratamiento de elección en pacientes con CA leve-moderada; no obstante, por diversas razones, como son edad avanzada, comorbilidad significativa, terapia anticoagulante, coledocolitiasis simultánea, rechazo a la cirugía o decisión del cirujano, se realiza tratamiento antibiótico sin intervención. En los pacientes con CA grave sin comer bilidad significativa se indica como tratamiento iniciâl la colecistectomía, mientras que en aquellos con comorbilidad importante se indica drenaje urgente mediante colecistostomía ecoguiada. Los pacientes coñ̈CA grave no intervenidos y con buena respuesta al $\bar{t}$ ratamiento médico inicial pueden completar el trätamiento antibiótico sin realizar colecistostomía. En⿳亠丷厂 pacientes con CA moderada sin mejoría clínica fambién se puede plantear la colecistostomía. En algưnos pacientes seleccionados con coledocolitiasis se realizó de forma urgente esfinterotomía endoscópica para extracción de cálculos y posteriormente colecistectomía.

\section{Tratamiento quirúrgico}

La CL la realizamos con el paciente en posiçión francesa, acceso mediante técnica de Hasson umbilical y tres puertos accesorios, manteniendo una presión de $12 \mathrm{mmHg}$. En caso de conversión o contraindicación a la laparoscopia se realiza lapărotomía subcostal. La colecistectomía subtotal o parcial se ha considerado un recurso válido en caso de dificultad tanto en cirugía laparoscópica como en caso de conversión, y no se ha incluido en el análisis $L a$ colocación de drenaje queda a criterio del cirujăno. En ocasiones, la intervención fue realizada por residentes mayores, pero siempre bajo la supervisión de un adjunto experimentado. El tratamiento posopẹratorio incluye antibiótico según protocolo, analgesia con paracetamol y metamizol, omeprazol y protócolo de enfermedad tromboembólica. La ingesta oral se 
Tabla 3. Resultados de la colecistitis aguda según los grupos de edad

\begin{tabular}{|c|c|c|c|c|c|c|}
\hline $\begin{array}{l}\text { Grupo de edad } \\
\text { (años) }\end{array}$ & Pacientes & $\begin{array}{l}\text { Comorbilidad } \\
\text { significativa }\end{array}$ & $\begin{array}{l}\text { Grado III } \\
\text { (grave) }\end{array}$ & Colecistectomía & $\begin{array}{c}\text { Complicación } \\
\text { Dindo-Clavien } \geq \text { II }\end{array}$ & Mortalidad \\
\hline $10-20$ & $4(0.4)$ & $0(0)$ & $0(0)$ & $2(50)$ & $0(0)$ & 0 (⿸广户्丶 \\
\hline $20-30$ & $21(2.1)$ & $2(9.5)$ & $1(4.8)$ & $19(90.5)$ & $4(19.0)$ & 0 (ब्)े \\
\hline $30-40$ & $52(5.2)$ & $1(1.9)$ & $1(1.9)$ & 45 (86.5) & $5(9.6)$ & 0 (0) \\
\hline $40-50$ & $73(7.3)$ & $2(2.7)$ & $0(0)$ & $57(78.1)$ & $4(5.5)$ & $0(0)$ \\
\hline $50-60$ & $94(9.4)$ & $14(14.9)$ & $7(7.4)$ & 67 (71.3) & $10(10.6)$ & $1(1$. \\
\hline $60-70$ & $155(15.5)$ & $44(28.4)$ & $12(7.7)$ & $108(69.7)$ & $15(9.7)$ & $2(\overline{13})$ \\
\hline $70-80$ & $251(25.1)$ & $107(42.6)$ & $19(7.6)$ & $165(65.7)$ & 32 (12.7) & $4(1.6)$ \\
\hline $80-90$ & 296 (29.6) & $160(54.0)$ & $47(15.8)$ & $114(38.5)$ & $49(16.5)$ & $11\left(\frac{317}{0}\right)$ \\
\hline$>90$ & $52(5.2)$ & 33 (63.5) & $6(11.5)$ & $5(9.61)$ & $7(13.5)$ & $2(3,8)$ \\
\hline Global & 998 & $363(36.4)$ & $93(9.3)$ & $582(58.3)$ & $126(12.6)$ & $20(20)$ \\
\hline
\end{tabular}

Todos los datos en $n(\%)$

reinicia a las 6 horas de la intervención con líquidos y aumento progresivo en función de la tolerancia.

\section{Tratamiento médico}

Los pacientes ingresados que no son intervenidos reciben el mismo tratamiento. La ingesta oral en los pacientes sin náuseas ni vómitos se inicia al llegar a planta con líquidos y se aumenta progresivamente en función de la tolerancia. En caso de colecistostomía ecoguiada se interrumpe la ingesta 6 horas antes de la prueba, y se reinicia tras esta.

\section{Estudio estadístico}

Al tratarse de un estudio observacional no se realizó una estimación formal del tamaño de la muestra. Las variables cuantitativas se expresaron mediante media y desviación estándar y se compararon con test t de Student o ANOVA. Las variables cuantitativas se expresaron mediante frecuencia y porcentajes, y se compararon mediante prueba de ji al cuadrado o prueba exacta de Fisher. En el estudio multivariante se introdujeron las variables con $p<0.20$ clínicamente significativas, y se realizó un análisis de regresión logística. El nivel de significación estadística se estableció en $p<0.05$. Se empleó SPSS versión 19.0 (IBM Co, Armonk, NY, USA).

\section{Resultados}

Entre junio de 2010 y diciembre de 2015 se incluyeron 998 pacientes con CA, de los cuales eran varones 574 (57,5\%), la edad media era de 70,0 $\pm 16,9$ años, y se distribuyeron en 338 (34\%) CA leves, $\frac{}{567}$ (57\%) CA moderadas y $93(9 \%)$ CA graves.

La tabla 3 presenta de manera descriptiva la evolución de los resultados según la edad divididâ en décadas. Se observa cómo en función del aumento de la edad se incrementan de forma progresiva y casi continua tanto el número de pacientes como la presencia de comorbilidad, CA grave, complicaciones y mortalidad, mientras que, de forma inversa, el trătamiento quirúrgico desciende.

\section{Características de la serie}

En la tabla 4 se exponen los datos globales y divididos según la gravedad. Aunque en la práctica totalidad de los parámetros se observan diferenêias significativas, los datos sugieren que dichas diferencias las puede marcar la CA grave. Así, al comparar la CA leve y la CA moderada no existen diferencias en lo que respecta a demografía, antecedentes y,comorbilidad, pero se mantienen en cuanto al tratamiento recibido $(p=0.000)$, la duración del tratamiênto antibiótico $(p<0.0001)$, la estancia media $(p<0.0001)$, las complicaciones $(p<0.0001)$, la mortalî́dad $(p<0.0001)$ y los reingresos $(p=0.044)$.

El tratamiento inicial fue antibioticoterapia en 404 (40.5\%) pacientes, de los cuales precisaron resceate quirúrgico 15 (3.5\%): 11 (5\%) con CA moderada 4 (11\%) con CA grave; y rescate mediante colecistostomía $8(2 \%)$. En la figura 1 se analizan las causas por las que no se realizó tratamiento quirúrgico. 
Tabla 4. Características de la serie, globales y en función del grado de colecistitis aguda

\begin{tabular}{|c|c|c|c|c|c|}
\hline Características & $\begin{array}{l}\text { Global } \\
(n=998)\end{array}$ & $\begin{array}{l}\text { Grado I (leve) } \\
\quad(n=338)\end{array}$ & $\begin{array}{l}\text { Grado II (moderada) } \\
\qquad(\mathrm{n}=567)\end{array}$ & $\begin{array}{l}\text { Grado III (grave) } \\
\qquad(\mathrm{n}=93)\end{array}$ & 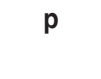 \\
\hline Edad* $^{*}$ & $70.0 \pm 17$ & $68.7 \pm 18$ & $69.9 \pm 17$ & $77.5 \pm 12$ & $<0.001$ \\
\hline Pacientes > 80 años & $348(34.9)$ & $106(31.4)$ & $189(33.3)$ & $53(57)$ & 0.001 \\
\hline Sexo masculino & $574(57.5)$ & $187(55.3)$ & $334(58.9)$ & $53(57)$ & 0.586 \\
\hline Diabetes & $266(26.7)$ & $86(25.4)$ & $146(25.7)$ & $34(37)$ & 0.721 \\
\hline Terapia anticoagulante & $128(12.8)$ & $39(11.5)$ & $59(10.4)$ & $30(32)$ & 0.001 \\
\hline Comorbilidad significativa & $363(36.4)$ & $105(31.1)$ & $192(33.9)$ & $66(71)$ & $<\overline{\theta 0} 01$ \\
\hline Antecedentes biliares & $81(8.1)$ & $25(7.4)$ & $41(7.2)$ & $15(16)$ & 0.072 \\
\hline Coledocolitiasis & $56(5.6)$ & $22(6.5)$ & $26(4.6)$ & $8(9)$ & 0.024 \\
\hline $\begin{array}{l}\text { Síntomas hasta el ingreso* } \\
\text { Tratamiento recibido }\end{array}$ & $3.3 \pm 4$ & $1.5 \pm 1$ & $4.1 \pm 4$ & $3.8 \pm 4$ & $\begin{array}{l}0.001 \\
\frac{0.00}{0} \\
\frac{2}{6}\end{array}$ \\
\hline $\begin{array}{l}\text { Antibioticoterapia } \\
\text { Colecistostomía } \\
\text { Colecistectomía }\end{array}$ & $\begin{array}{c}381(38.2) \\
35(3.5) \\
582(58.3)\end{array}$ & $\begin{array}{c}184(54.4) \\
0(0) \\
154(45.6)\end{array}$ & $\begin{array}{c}182(32.1) \\
17(3.0) \\
368(64.9)\end{array}$ & $\begin{array}{l}15(16) \\
18(19) \\
60(64)\end{array}$ & $\begin{array}{l}0.001 \\
0.001 \\
0.001\end{array}$ \\
\hline Duración del antibiótico* & $7.2 \pm 4$ & $6.3 \pm 3$ & $7.4 \pm 4$ & $9.0 \pm 5$ & 0.001 \\
\hline Estancia hospitalaria* & $5.7 \pm 6$ & $4.2 \pm 3$ & $5.5 \pm 4$ & $12.5 \pm 13$ & 0.001 \\
\hline Complicación Dindo-Clavien $\geq$ II & $126(12.6)$ & $12(3.6)$ & $69(12.2)$ & $45(49)$ & \\
\hline Mortalidad $^{\dagger}$ & $20(2.0)$ & $0(0)$ & $3(0.5)$ & $17(18)$ & $0: 001$ \\
\hline Reingresos $^{\dagger}$ & $29(2.9)$ & $4(1.2)$ & $21(3.7)$ & $4(5)$ & $0: 042$ \\
\hline Recidiva de colecistitis ${ }^{\ddagger}$ & $83(20.3)$ & $34(18.5)$ & $43(21.6)$ & $6(23)$ & 0.241 \\
\hline Otra complicación biliarł & $44(10.8)$ & $20(10.9)$ & $22(11.1)$ & $2(8)$ & 0.832 \\
\hline \multicolumn{5}{|c|}{$\begin{array}{l}\text { Media } \pm \text { desviación estándar en días. } \\
+ \text { † } 30 \text { días. } \\
\text { "En pacientes no operados. } \\
\text { Excepto los señalados con asterisco, todos los datos en n (\%). }\end{array}$} & 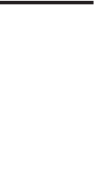 \\
\hline
\end{tabular}

Finalmente, del total de la serie se operaron 582 (58\%) pacientes (Tabla 5), y se observan diferencias en todas las variables perioperatorias y postoperatorias en función del grado. No hubo diferencias en cuanto al número de fístulas biliares, con un total de 12 (2.0\%), de las que tres se resolvieron de manera conservadora, cuatro mediante reintervención y cinco mediante Colangiografía Retrógrada Endoscópica Percutánea (CREP).

\section{Factores de riesgo de morbilidad y mortalidad}

Hemos analizado qué factores se han podido asociar con complicaciones y mortalidad en los pacientes con CA (Tabla 6). Como factores de riesgo independiente de mortalidad encontramos la CA grave y la comorbilidad significativa. Factores de riesgo independientes de complicaciones Dindo-Clavien $\geq$ II son la CA grave y la conversión a cirugía abierta.
La CA grave ha sido un factor de riesgo importânte en pacientes tanto no operados como operados. $\overline{-} A s i ́$, en los pacientes no intervenidos la mortalidad fue nula en las CA leves y moderadas, frente a $7(21 \%)$ faltecimientos en las CA graves ( $p<0.0001)$; las complicaciones Dindo-Clavien $\geq$ II se presentaron en 19 (5\%) vs. $12(36 \%)$, respectivamente $(p<0.0001)$. Enlos pacientes operados, la mortalidad fue de $3(0.6 \%)$ en las CA leves y moderadas, y de $10(17 \%)$ en las $\mathrm{CA}$ graves ( $p<0.0001$ ); las complicaciones Dindo-Clàvien $\geq$ II se presentaron en $62(12 \%)$ y $33(56 \%)$, respectivamente $(p<0.0001)$.

\section{Factores de riesgo de presentar CA grave}

Dada su importancia en el pronóstico de los pacientes, analizamos qué factores han podido favorecer la CA grave (Tabla 7). En el análisis multivariante fueron factores independientes la edad mayor de 80 años 


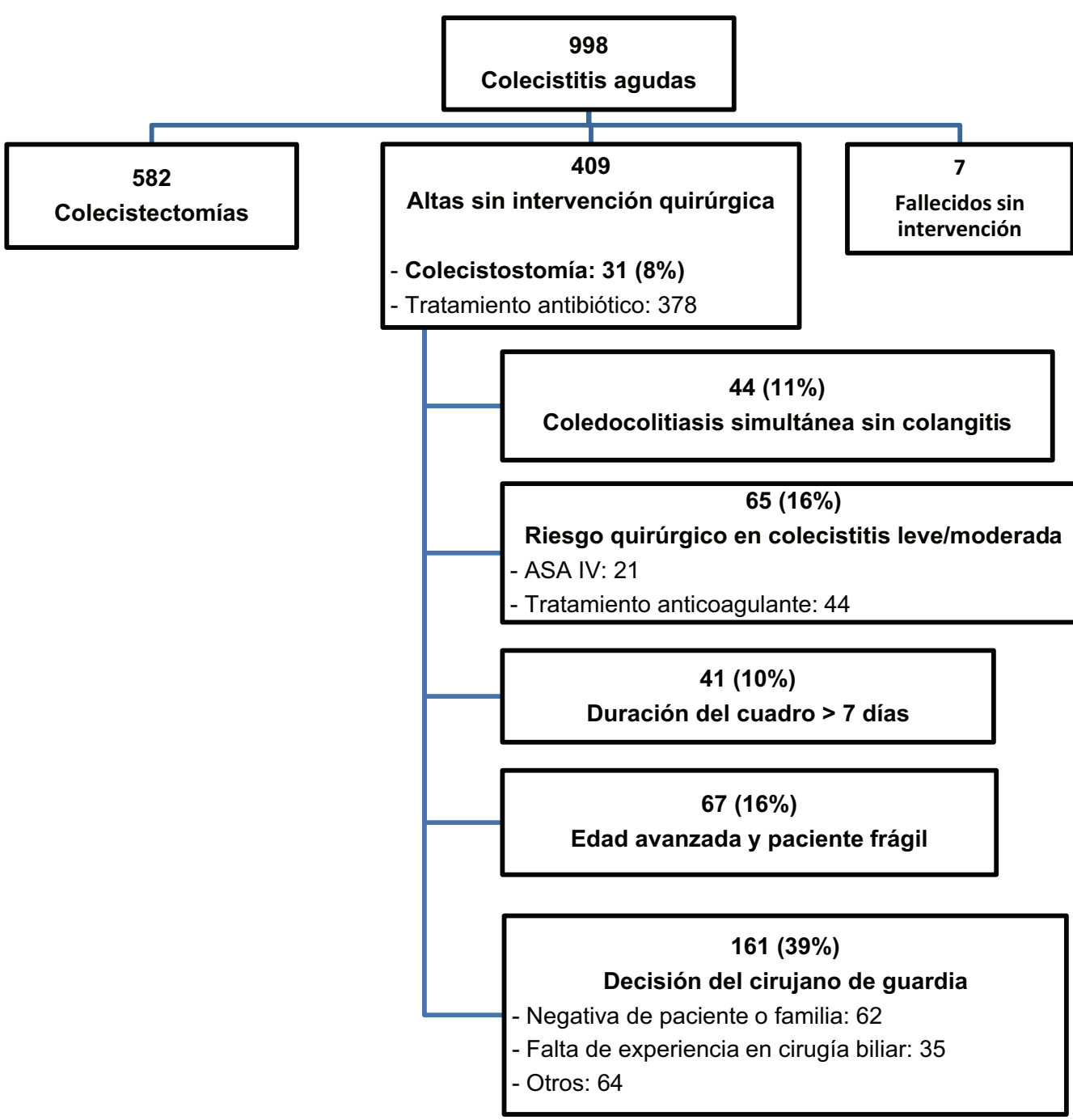

Figura 1. Causas por las que no se realizó tratamiento quirúrgico.

(odds ratio $[\mathrm{OR}]=1.8$; intervalo de confianza del 95\% [IC 95\%]: 1.1-2.8; $p=0.011$ ), los antecedentes biliares $(\mathrm{OR}=2,06$; IC 95\%: 1.1-3.8; $p=0.025)$ y la comorbilidad significativa (OR $=4.16$; IC 95\%: 2.5-6.7; $p<0.0001$ ).

\section{Discusión}

La CA es una de las urgencias quirúrgicas más frecuentes en nuestro medio, como demuestra el hecho de haber incluido casi mil casos en menos de 6 años. El incremento en su incidencia viene parejo al aumento de la esperanza de vida en nuestro país, la mayor del mundo ${ }^{27}$ : más de la mitad de los casos corresponden a pacientes mayores de 70 años $^{28}$. Pero esto no es un mero dato demográfico, pues hemos comprobado que a la par que se va elevando la edad se incrementa la comorbilidad y aumenta el número de CA graves, lo que se traduce en un aumento de la morbilidad y de la mortalidad.

La edad avanzada (cuya consideración ha ido pasando de los 65 a los 80-90 años) $)^{15-18,28}$ y la comorbilidad asociada ${ }^{18,29,30}$ han sido considerados 힝s principales factores de riesgo de complicaciones, $\frac{\bar{c}}{\mathrm{Co}}$ siderando en ocasiones determinados hallazgos ānalíticos, radiológicos o quirúrgicos ${ }^{12-14,31}$, aunque sin tuna definición unánime de gravedad, lo que impedía cómparar los diversos estudios. Otros factores analizados al valorar el riesgo de la colecistectomía son los días de clínica hasta la intervención, pero la falta de hom̄ogeneidad en la medición (algunos estudios to ⿳亠丷厂̆an como punto de partida el día 0 de clínica y otros el momento de ingreso del paciente) hace difícil la comparación y el establecimiento de conclusiones,7,9. 
Cirugía y Cirujanos. 2021;89(1)

Tabla 5. Resultados de los pacientes con colecistitis aguda intervenida quirúrgicamente

\begin{tabular}{|c|c|c|c|c|c|}
\hline Características & $\begin{array}{l}\text { Pacientes intervenidos } \\
\qquad(\mathrm{n}=582)\end{array}$ & $\begin{array}{l}\text { Grado I (leve) } \\
\qquad(n=154)\end{array}$ & $\begin{array}{l}\text { Grado II (moderada) } \\
\qquad(\mathrm{n}=368)\end{array}$ & $\begin{array}{l}\text { Grado III (grave) } \\
\qquad(n=60)\end{array}$ & 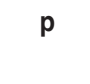 \\
\hline Comorbilidad significativa & $162(27.8)$ & $29(18.8)$ & $91(24.7)$ & $42(70)$ & 0.001 \\
\hline Colecistectomía precoz & $548(94.2)$ & $151(98.1)$ & $344(93.5)$ & $53(88)$ & 0.016 \\
\hline Colecistectomía laparoscópica & $545(93.6)$ & $152(98.7)$ & $341(92.7)$ & $52(87)$ & 0.002 \\
\hline Conversión a cirugía abierta & $41(7.5)$ & $1(0.7)$ & $31(9.1)$ & $9(17)$ & $=0.001$ \\
\hline \multicolumn{5}{|l|}{ Hallazgos } & ت্ত্রে \\
\hline Edematosa & $197(34.3)$ & $116(76.8)$ & $70(19.2)$ & $11(18)$ & $0=001$ \\
\hline Gangrenosa & $289(50.3)$ & $31(20.5)$ & $228(62.6)$ & $30(50)$ & $\frac{0}{3}$ \\
\hline Perforada/coleperitoneo & $89(15.5)$ & $4(2.6)$ & $66(18.2)$ & $19(32)$ & $\frac{2}{0}$ \\
\hline Colocación de drenaje & $347(59.7)$ & $51(33.1)$ & $253(68.9)$ & $43(78)$ & 0.001 \\
\hline Fístula biliar & $12(2.0)$ & $2(1.3)$ & $7(1.9)$ & $3(5)$ & 0.251 \\
\hline Estancia hospitalaria* & $5.9 \pm 7$ & $3.7 \pm 4$ & $5.5 \pm 5$ & $13.5 \pm 14$ & +0.001 \\
\hline Complicación Dindo-Clavien $\geq$ II & $95(16.4)$ & $10(6.5)$ & $52(14.1)$ & $33(56)$ & 0.001 \\
\hline Mortalidad ${ }^{\dagger}$ & $13(2.2)$ & $0(0)$ & $3(0.8)$ & $10(17)$ & $0=01$ \\
\hline Reingresos ${ }^{\dagger}$ & $20(3.5)$ & $4(2.6)$ & $12(3.3)$ & $4(8)$ & $0 . \frac{192}{0} 2$ \\
\hline \multicolumn{5}{|c|}{$\begin{array}{l}\text { *Media } \pm \text { desviación estándar en días. } \\
\text { †A } 30 \text { días. } \\
\text { Excepto los señalados con asterisco, todos los datos en n (\%). }\end{array}$} & 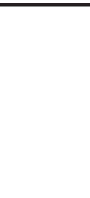 \\
\hline
\end{tabular}

La falta de criterios uniformes para el diagnóstico y la gravedad de la CA promovió la publicación en $2007^{19}$ (revisada en $2013^{20}$ y $2018^{21}$ ) de las Guías de Tokio. Estas guías, más allá de servir de ayuda a la toma de decisiones en la práctica clínica diaria, permiten una homogeneidad de criterios y definiciones que harán más fácil la posterior comparación de estudios y resultados, aunque inicialmente no parecieran mejorar los resultados de los pacientes con $\mathrm{CA}^{32}$.

Basándonos en estas guías planteamos nuestro estudio: todos los pacientes incluidos cumplieron criterios diagnósticos y posteriormente fueron clasificados por gravedad. El grupo de CA moderada ha sido el más numeroso al incluir como criterio la sintomatología de más de 72 horas al iniciar el tratamiento (en ocasiones pacientes con sintomatología leve y poca afectación analítica). Esto ha podido influir en que CA leves y moderadas no presenten diferencias en cuanto a demografía, antecedentes y comorbilidad ${ }^{33}$, aunque ambas están claramente diferenciadas respecto a la $\mathrm{CA}$ grave. Sin embargo, los tres grupos guardan claras diferencias en lo que respecta al tratamiento aplicado y los resultados.

Las Guías de Tokio dan cierta libertad para seleccionar el tratamiento ${ }^{34}$, siendo nuestra primera opción el tratamiento quirúrgico, debido a la alta tasa de recidivas en los pacientes no operados (en torno al $20 \%$ independientemente del grado de la CA) y la peor evolución de la recidiva de la CA respecto al primer episodio $^{8,35}$. El grado ha influido en los resultado de los pacientes intervenidos: a mayor grado hemos servado más complejidad en la intervención, mášnecesidad de drenajes y un mayor índice de conversiones, asociando una estancia posoperatoria más larga y.con mayor morbilidad y mortalidad, sobre todo en el grupo de CA grave. A pesar de ello, las complicaciones de tipo iatrogénico sobre la vía biliar no han presentado diferencias en función del grado, lo que sugiere quie la morbimortalidad viene determinada más por la afectación sistémica de la CA que por las dificultades técnicas de la intervención.

La aplicación de las Guías de Tokio a nuestros spacientes nos ha permitido categorizar de forma efectiva el grado y, según este, aplicar el tratamiento más ădecuado. Los resultados de las CA leves han sido excelentes independientemente del tratamiento aplicădo. Buenos han sido también los resultados en las $\mathrm{CA}$ moderadas, pero son pacientes de mayor riesgo y ĕssto deberá ser tenido en cuenta al plantear el tratamiento. Respecto a las CA graves, se trata de un grupo de pacientes de alto riesgo con elevadas morbilidad y mortalidad que con frecuencia requerirán cuidados intensivos y un tratamiento inmediato e individualizâdo, en especial en el caso de los mayores de 80 años 28 . 
Tabla 6. Factores de riesgo de mortalidad

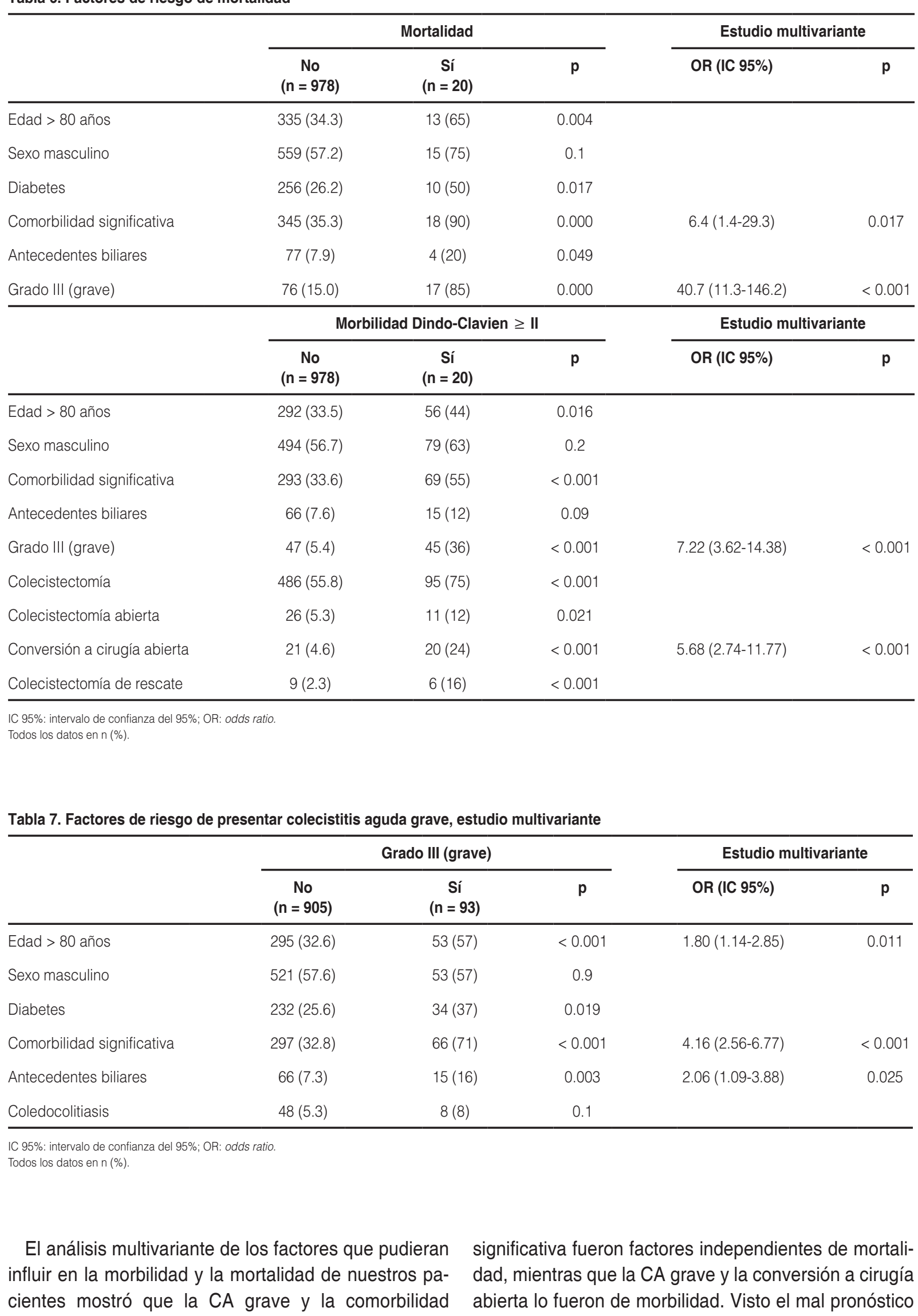


de los pacientes con CA grave, analizamos qué factores pueden determinar su aparición, y hallamos como factores independientes la comorbilidad significativa, la presencia de antecedentes biliares y la edad mayor de 80 años. A pesar del riesgo que supone en los pacientes intervenidos la comorbilidad significativa (ASA IIIIV), nuestros resultados en la CA leve y moderada justifican la intervención, dado el riesgo de recidiva en caso de no intervención; en la CA grave podría ser más beneficioso el drenaje radiológico, salvo si hay coleperitoneo o si no puede realizarse la colecistostomía.

Nuestra experiencia coincide con una encuesta a cirujanos españoles': aun queriendo operar todas las CA, cuesta superar el $60 \%$ de pacientes intervenidos. El ingreso a cargo de la unidad hepatobiliar y no del cirujano de guardia puede aumentar esta cifra, además de la seguridad del paciente en casos complejos. Aunque nos marcamos como límite teórico para la intervención el séptimo día tras el inicio de los síntomas, el $94 \%$ de las intervenciones se realizaron en las primeras 48 horas, lo que ha podido influir positivamente en nuestros resultados.

En conclusión, proponemos la CL urgente en el momento del ingreso como tratamiento de elección en la CA leve y moderada, independientemente de la edad del paciente ${ }^{28}$ (salvo en casos en los que haya contraindicación anestésica, otras situaciones como coledocolitiasis concomitante, o CA de más de 7 días de evolución), basándonos en nuestros buenos resultados en los pacientes intervenidos y teniendo presente que el índice de recidiva de la CA es superior al $20 \%$ y con peores resultados que en el primer episodio ${ }^{35}$. Los pacientes con CA grave presentan una alta morbimortalidad y deberían ser atendidos, o en su caso trasladados, a centros que cuenten con recursos adecuados y cirujanos con experiencia en cirugía biliar.

\section{Conflicto de intereses}

Los autores declaran no tener ningún conflicto de intereses.

\section{Financiamiento}

Los autores no recibieron patrocinio para llevar a cabo este artículo. La presente investigación no ha recibido ayudas específicas provenientes de agencias del sector público, del sector comercial ni de entidades sin ánimo de lucro.

\section{Responsabilidades éticas}

Protección de personas y animales. Los autores declaran que para esta investigación no se han realizado experimentos en seres humanos hi ${ }^{\bar{E}} \mathrm{en}$ animales.

Confidencialidad de los datos. Los autores deelaran que han seguido los protocolos de su centro: de trabajo sobre la publicación de datos de pacientes.

Derecho a la privacidad y consentimiento informado. Los autores declaran que en este artículöno aparecen datos de pacientes.

\section{Bibliografía}

1. Badia JM, Nve E, Jimeno J, Guirao X, Figueras J, Arias-Diaz J. Sưfgical management of acute cholecystitis. Results of a nation-wide sürvey among Spanish surgeons. Cir Esp 2014;92:517-24.

2. Campanile FC, Pisano M, Coccolini F, Catena F, Agresta F, Ansaloni L. Acute cholecystitis: WSES position statement. World J Emerg Surg. 2014:9:58.

3. Gurusamy KS, Davidson C, Gluud C, Davidson BR. Early versus delayed laparoscopic cholecystectomy for people with acute cholecystitis? Cochrane Database Syst Rev. 2013;(6):CD005440.

4. Gutt CN, Encke J, Köninger J, Harnoss JC, Weigand K, KipfmüتTer K, et al. Acute cholecystitis: early versus delayed cholecystectomy, amulticenter randomized trial (ACDC study, NCT00447304). Ann +Surg. 2013;258:385-93.

5. Cao AM, Eslick GD, Cox MR. Early cholecystectomy is superior to-delayed cholecystectomy for acute cholecystitis: a meta-analysis. J Gastrointest Surg. 2015;19:848-57.

6. Calero García P, Ruiz Tovar J, Sanjuanbenito Dehesa A, Calero Amaro A, Díez Tabernilla M, Latorre Fragua R, et al. Acute cholecystitis: is it still justified to delay surgery? Cir Esp. 2010;88:92-6.

7. Agrawal R, Sood KC, Agarwal B. Evaluation of early versus delayed laparoscopic cholecystectomy in acute cholecystitis. Surg Res Pract. 2015;2015:349801.

8. De Mestral C, Rotstein OD, Laupacis A, Hoch JS, Zagorski B, Nathens AB. A population-based analysis of the clinical course of 10,304 patients with acute cholecystitis, discharged without cholecystectomy. J Trauma Acute Care Surg. 2013;74:26-30.

9. Haltmeier T, Benjamin E, Inaba K, Lam L, Demetriades D. Early verrsus delayed same-admission laparoscopic cholecystectomy for acute-cholecystitis in elderly patients with comorbidities. J Trauma Acute Care Surg. 2015;78:801-7.

10. Reyes ML, Milanes JA, Ruiz JA, Lafuente del R, Valdes J, Moreno LS, et al. Development of the surgical approach to acute cholecystitis in an emergency surgical unit. Cir Esp. 2012;90:186-90.

11. Riall TS, Zhang D, Townsend CM, Jr., Kuo YF, Goodwin JS. Failure to perform cholecystectomy for acute cholecystitis in elderly patients is associated with increased morbidity, mortality, and cost. J Am Coll@ $\mathbf{S}$ urg. 2010;210:668-77.

12. Kim JH, Kim JW, Jeong IH, Choi TY, Yoo BM, Kim JH, et al. Surgical outcomes of laparoscopic cholecystectomy for severe acute cholecystitis. J Gastrointest Surg. 2008;12:829-35.

13. Merriam LT, Kanaan SA, Dawes LG, Angelos P, Prystowsky JB, Rege RV, et al. Gangrenous cholecystitis: analysis of risk factors and experience with laparoscopic cholecystectomy. Surgery. 1999;126:68085. Discussion 685-6.

14. Weiss CA 3rd, Lakshman TV, Schwartz RW. Current diagnosis and treatment of cholecystitis. Curr Surg. 2002;59:51-4.

15. Coenye KE, Jourdain S, Mendes da Costa P. Laparoscopic cholecystectomy for acute cholecystitis in the elderly: a retrospective study. Hepatogastroenterology. 2005;52:17-21.

16. Sanchez-Beorlegui J, Soriano P, Monsalve E, Moreno N, Cabezali R, Navarro A. Laparoscopic cholecystectomy in octogenarian patients. A comparative study between two geriatric population groups. Cir Esp. 2009;85:246-51.

17. Bueno J, Vaque J, Herrero C, Castillo E, Carbonell F, Baquero R, et al. Acute cholecystitis and laparoscopic cholecystectomy in the elderity. Cir Esp. 2007;81:213-7.

18. Dubecz A, Langer M, Stadlhuber RJ, Schweigert M, Solymosi N, Feith M, et al. Cholecystectomy in the very elderly - is 90 the new 70 ? J Gastrointest Surg. 2012;16:282-5. 
19. Hirota M, Takada T, Kawarada Y, Nimura Y, Miura F, Hirata K, et al. Diagnostic criteria and severity assessment of acute cholecystitis: Tokyo Guidelines. J Hepatobiliary Pancreat Surg. 2007;14:78-82.

20. Yokoe M, Takada T, Strasberg SM, Solomkin JS, Mayumi T, Gomi H, et al. TG13 diagnostic criteria and severity grading of acute cholecystitis (with videos). J Hepatobiliary Pancreat Sci. 2013;20:35-46.

21. Yokoe M, Hata J, Takada T, Strasberg S, Asbun H, Wakabayashi G, et al. Tokyo Guidelines 2018: diagnostic criteria and severity grading of acute cholecystitis (with videos). J Hepatobiliary Pancreat Sci. 2018;25:41-54.

22. Dindo D, Demartines N, Clavien PA. Classification of surgical complications: a new proposal with evaluation in a cohort of 6336 patients and results of a survey. Ann Surg. 2004;240:205-13.

23. Escartin A, Mias MC, Gonzalez M, Cuello E, Pinillos A, Muriel P, et al. Home hospitalization for surgical and conservative treatment of acute calculous cholecystitis. Surgical Practice. 2018;22:52-9.

24. Kanafani Z, Khalifé N, Kanj S, Araj G, Khalifeh M, Sharara A. Antibiotic use in acute cholecystitis: practice patterns in the absence of evidence-based guidelines. J Infect. 2005;51:128-34.

25. Guirao X, Arias J, Badia JM, Garcia-Rodriguez JA, Mensa J, Alvarez-Lerma $F$, et al. Recommendations in the empiric anti-infective agents of intra-abdominal infection. Cir Esp. 2010;87:63-81.

26. Yoshida M, Takada T, Kawarada Y, Tanaka A, Nimura Y, Gomi H, et al. Antimicrobial therapy for acute cholecystitis: Tokyo Guidelines. J Hepatobiliary Pancreat Surg. 2007;14:83-90.

27. Health at a Glance 2017. OECD Indicators. Disponible en: https://doi. org/10.1787/health_glance-2017-en
28. Escartín A, González M, Cuello E, Pinillos A, Muriel P, Merichal M, et al. Acute cholecystitis in very elderly patients: Disease management, outcomes and risk factors for complications. Surg Res Pract. Z2019; 2019:9709242.

29. Loozen CS, van Ramshorst B, van Santvoort HC, Boerma D. Acute cholecystitis in elderly patients: a case for early cholecystectomy. IVisc Surg. 2018;155:99-103.

30. Nikfarjam M, Yeo D, Perini M, Fink MA, Muralidharan V, Starkey G.et al. Outcomes of cholecystectomy for treatment of acute cholecystitis in octogenarians. ANZ J Surg. 2014;84:943-8.

31. Real-Noval H, Fernández-Fernández J, Soler-Dorda G. Predicting factors for the diagnosis of gangrene acute cholecystitis. Cir Cir. 2019;87:443-9.

32. Lee SW, Yang SS, Chang CS, Yeh HJ. Impact of the Tokyo guidelines on the management of patients with acute calculous cholecystitis. $J$ Gastroenterol Hepatol. 2009;24:1857-61.

33. Loozen CS, Blessing MM, Van Ramshorst B, Van Santvoort HC, Boerma D. The optimal treatment of patients with mild and moderate acute cholecystitis: time for a revision of the Tokyo Guidelines. Surg Endosc. 2017;31:3858-63.

34. Miura F, Takada T, Strasberg SM, Solomkin JS, Pitt HA, Gouma DJ, et al. TG13 flowchart for the management of acute cholangitis and cholecystitis. J Hepatobiliary Pancreat Sci. 2013;20:47-54.

35. Escartín A, González M, Pinillos A, Cuello E, Muriel P, Tur J, ęt al. Failure to perform index cholecystectomy during acute cholecystitis results in significant morbidity for patients who present with recurfence. HPB (Oxford). 2019;21:876-82. 